\title{
Harmonic Operation of a Free-Electron Laser
}

\author{
P. E. Latham, B. Levush, and T. M. Antonsen, Jr. \\ University of Maryland, College Park, Maryland 20742 \\ N. Metzler \\ Nuclear Research Center-Negev, P.O. Box 90001, Beer-Sheva, Israel
}

(Received 13 September 1990)

\begin{abstract}
Harmonic operation of a free-electron-laser amplifier is studied. The key issue investigated here is suppression of the fundamental. For a tapered amplifier with the right choice of parameters, it is found that the presence of the harmonic mode greatly reduces the growth rate of the fundamental. A limit on the reflection coefficient of the fundamental mode that will ensure stable operation is derived. The relative merits of tripling the frequency by operating at the third harmonic versus decreasing the wiggler period by a factor of 3 are discussed.
\end{abstract}

PACS numbers: $42.55 . \mathrm{Tb}$

A free-electron laser (FEL) produces coherent radiation when an electron beam passes through a spatially periodic magnetic field (called a wiggler). The frequency of the amplified radiation is determined by the condition that the Doppler-shifted frequency of the radiation, viewed in the beam frame, coincides with a harmonic of the Doppler-shifted frequency of the wiggler. As a result, the frequency scales as the harmonic number times the square of the beam voltage divided by the wiggler period.' Because of the favorable scaling with voltage, FELs show promise for operating at high frequencies. However, there are technological limits on attainable voltages, and the gain falls off rapidly with decreasing wiggler period (we give explicit scaling of gain versus frequency below). Thus, to reach ultrahigh frequencies it may be advantageous to operate at a harmonic of the fundamental frequency.

The existence of higher harmonics in the emission spectrum of a FEL was predicted in a number of theoretical publications, ${ }^{1}$ and has been measured in several experiments. $^{2}$ However, these experiments were designed to operate in the fundamental mode; harmonic generation, which was weak, was merely a by-product associated with bunching of the electron beam in a linearly polarized wiggler. It would be desirable to construct a FEL in which the harmonic mode, rather than the fundamental, dominated. The primary difficulty with such an approach is that the fundamental mode has a larger growth rate than the harmonic, and tends to dominate in the nonlinear regime. This is an especially serious problem in an oscillator as experiments run for many growth times. In an amplifier with an injected signal, on the other hand, it is only necessary to reduce the growth rate of the fundamental enough so that its amplitude is still small at the end of the device. For this reason we investigate an amplifier in which a signal is injected at the harmonic frequency and the fundamental is allowed to grow from noise. The goal of our analysis is to understand how the presence of the harmonic effects the growth rate of the fundamental.

We consider a one-dimensional model in which the ra- diation undergoes spatial amplification as it interacts with an electron beam in a wiggler magnetic field. The vector potential for the wiggler field is written

$$
\mathbf{A}_{\text {wiggle }}=A_{w}(z) \cos \phi(z) \hat{\mathbf{x}},
$$

where $d \phi / d z=k_{w}(z)$, and the amplitude $A_{w}$ and wiggler wave number $k_{w}$ are specified functions of $z$. The radiation field is composed of a superposition of plane waves with slowly varying amplitudes and phases. For simplicity, we neglect dispersion; i.e., the radiation field is independent of transverse position and its group velocity is $c$, the speed of light. With these simplifications, the $x$ component of the radiation field is expressed as

$$
A_{x}=\sum_{n} A_{n}(z, t) e^{i\left(k_{n} z-\omega_{n} t\right)}+\text { c.c. },
$$

where $\omega_{n}$ and $k_{n}$ are, respectively, the frequency and wave number of the $n$th harmonic, and $A_{n}(z, t)$ is a slowly varying function of its arguments. For a planer wiggler, which we consider here, only the odd harmonics $(n=1,3,5, \ldots)$ are present. In the absence of dispersion, the frequency and wave number of each harmonic are given by $\omega_{n}=n \omega_{0}$ and $k_{n}=n k_{0}$ with $\omega_{0} / k_{0}=c$. The fundamental frequency $\omega_{0}$ will be defined below.

In our analysis we consider just two terms in the sum on harmonic mode number [Eq. (1)]: the fundamental $(n=1)$ and the $s$ th harmonic $(n=s)$. We assume that the harmonic is injected at a single frequency, $s \omega_{0}$ with $s>1$ (here defining $\omega_{0}$ ), and thus $A_{s}$ is independent of time. The frequency $s \omega_{0}$ is chosen to correspond to the fastest-growing harmonic mode. The fundamental mode, on the other hand, consists of a superposition of waves with different frequencies:

$$
A_{1}(z, t)=\sum_{m} A_{1 m}(z) e^{-i m \Omega t} .
$$

Thus, the expression for the $x$ component of the radiation field becomes

$$
A_{x}=\sum_{m} A_{1 m}(z) e^{i\left(k_{0} z-\omega_{0} t-m \Omega t\right)}+A_{s}(z) e^{i s\left(k_{0} z-\omega_{0} t\right)}+\text { c.c. }
$$


The quantity $\Omega$ represents the frequency separation between the various fundamental signals. We choose $\Omega$ to be sufficiently small to resolve the spectral structure of the fundamental. The mode number $m$ takes on values between $m_{1}$ and $m_{2}$, chosen to include all modes with significant gain.

To simulate the FEL we find the current by numerically integrating the Lorentz-force equation for an ensemble of electrons, then use the current as a source term in Maxwell's equations to compute the amplitude of the radiation field. Each particle is specified by its beatwave phase $\psi$ defined by

$$
\psi \equiv \int^{z} \frac{d z^{\prime}}{c \beta_{z}\left(z^{\prime}, \phi\left(z^{\prime}\right)\right)}-\omega_{0} t
$$

where $z$ is the position of the particle at time $t$ and $\beta_{z}$ is the axial velocity of a resonant particle (resonant will be defined below) normalized to the speed of light:

$$
\beta_{z}(z, \phi) \equiv\left[1-\frac{1+a_{w}^{2}(z) \cos ^{2} \phi}{\gamma_{r}^{2}}\right]^{1 / 2} .
$$

[Recall that $d \phi / d z=k_{w}(z)$.] Here $a_{w}(z) \equiv q A_{w}(z) / m_{0} c^{2}$ is the wiggler parameter $\left(q_{0}\right.$ and $m_{0}$ are the particle charge and mass, respectively), and $m_{0} c^{2} \gamma_{r}$ is the resonant energy. Assuming that the $z$ dependence of $a_{w}$ and $k_{w}$ (tapering) is slow compared to a wiggler period, i.e., $d a_{w} / d z \ll k_{w} a_{w}$ and $d k_{w} / d z \ll k_{w}^{2}, \gamma_{r}$ is defined implicitly by demanding that the average velocity equal the beatwave phase velocity $\omega_{0} /\left[k_{0}+k_{w}(z)\right]$ :

$$
\bar{\beta}_{z}(z) \equiv\left[\int_{0}^{2 \pi} \frac{d \phi}{2 \pi} \beta_{z}^{-1}(z, \phi)\right]^{-1}=\frac{k_{0}}{k_{0}+k_{w}(z)} .
$$

It is convenient to work in normalized distance $\xi$ rather than $z$; to this end we introduce the scale factor $S(z)$,

$$
S(z) \equiv \frac{1}{\bar{\beta}_{z}^{2}(z)} \frac{\partial \bar{\beta}_{z}(z)}{\partial \gamma_{r}},
$$

and the gain length $L$ defined in terms of physical parameters as

$$
k_{0} L \equiv\left\{\frac{\gamma_{r}^{2}(0)}{4 \pi a_{w}^{2}(0) S(0)} \frac{k_{0}^{2} I_{0}}{J}\right\}^{1 / 3},
$$

with $J$ the current density averaged over the effective area of the radiation and $I_{0}=17045 \mathrm{~A}$. Then, $\xi$ is expressed in terms of $z, S$, and $L$ as

$$
\xi=\int^{z} \frac{d z^{\prime}}{L} \frac{S\left(z^{\prime}\right)}{S(0)} .
$$

Assuming that the particle energy stays close to the resonant energy $m_{0} c^{2} \gamma_{r}$, and the radiation amplitudes $A_{1 m}(z) e^{-i m \Omega t}$ and $A_{s}(z)$ are slowly varying functions of $z$ and $t$, the phase $\psi$ evolves according to

$$
\begin{aligned}
\frac{d^{2} \psi}{d \xi^{2}}= & 2 C_{1}(\xi) \sum_{m} \operatorname{Im}\left[a_{1 m}(\xi) e^{i \psi-i m \Omega_{0} \tau_{0}}\right] \\
& +2 s C_{s}(\xi) \operatorname{Im}\left[a_{s}(\xi) e^{i s \psi}\right]-\alpha(\xi),
\end{aligned}
$$

where initially $d \psi / d \xi=0$ and $\psi$ is uniformly distributed in the interval $[0,2 \pi]$. This equation is written in a Lagrangian frame ${ }^{3}$ moving at the phase velocity of the beat wave. Normalized distance $\xi$ rather than time is the independent variable, $\tau_{0} \equiv c t / L$ is now an initial condition representing the time at which an electron enters the interaction region, and $\Omega_{0} \equiv \Omega L / c$ is the normalized modulation frequency. The entrance times are uniformly distributed in the range $\left[0,2 \pi / \Omega_{0}\right]$. The deceleration term $\alpha(\xi)$, which appears because the equations of motion are written in a decelerating frame, and the coupling coefficients $C_{n}(\xi)$ depend on the type of tapering. Explicit expressions for these quantities will be given in Eqs. (12) and (13a) below, after we have specified the dependence of $\gamma_{r}(\xi)$ on $\xi$.

The normalized field amplitude $a_{n m}(\xi)$ is defined in terms of $A_{n m}(z)$ as

$$
a_{n m}(\xi)=\frac{a_{w}(0) S(0) k_{0}^{2} L^{2}}{\gamma_{r}(0) \beta_{z r}(0)} \frac{q A_{n m}(z) e^{-i m \Omega_{0} z / L}}{m_{0} c^{2}} .
$$

The wave equation for the slowly varying amplitude $a_{n m}(\xi)$ is written

$$
\begin{aligned}
{\left[i m \Omega_{0} \epsilon(\xi)+\frac{d}{d \xi}\right] } & a_{n m}(\xi) \\
& =-\frac{i}{2} \frac{C_{n}(\xi)}{n}\left\langle\left\langle e^{-i n \psi+i m \Omega_{0} \tau_{0}}\right\rangle\right\rangle,
\end{aligned}
$$

where the double angular brackets indicate an average over the initial phases and entrance times of the particles. The quantity $\epsilon(\xi) \equiv\left[\bar{\beta}_{z}^{-1}(\xi)-1\right] S(0) / S(\xi)$ is the normalized slippage parameter.

Equations (7) and (8) comprise our complete system. These equations depend on a number of parameters and functions: $a_{w}(\xi)$, the wiggler parameter, $m_{0} c^{2} \gamma_{r}(\xi)$, the resonant beam energy, and $L$, the gain length. Our goal is to choose these parameters so that the overall gain of the fundamental is as small as possible while still providing significant amplification of the third harmonic. In fact, a "good" harmonic FEL design is one in which the amplitude of the fundamental mode remains small enough that nonlinear effects are not important. Consequently, in our analysis we ignore all terms which are quadratic or higher order in the amplitude of the fundamental mode. The harmonic mode, on the other hand, is treated fully nonlinearly. When the harmonic becomes large enough that nonlinear effects are important, the growth rate of the fundamental is modified. An approximate dispersion relation for the fundamental mode can be derived if we assume that the amplitude and phase of the harmonic vary slowly compared to the growth time of the fundamental and most of the particles are trapped at the bottom of the harmonic beat-wave potential. Letting $a_{1 m} \sim e^{i \kappa_{m} \xi}$, the dispersion relation for $\kappa_{m}$ is

$$
\left[\kappa_{m}+m \Omega_{0} \epsilon(\xi)\right]\left[\kappa_{m}^{2}-\omega_{b}^{2}(\xi)\right]=-C_{1}^{2}(\xi) / 2,
$$

where the bounce frequency $\omega_{b}$ is defined by $\omega_{b}^{2} \equiv 2 s^{2}$ 
$\times\left|C_{s}(\xi) a_{s 0}(\xi)\right|$, with $a_{s 0}(\xi)$ the complex amplitude of the $s$ th harmonic.

Equation (9) describes the linear growth of the fundamental. In the absence of the harmonic $\left(\omega_{b}=0\right)$, it reduces to the familiar cubic equation for the spatial gain of a cold beam. In the presence of the harmonic $\left(\omega_{b} \neq 0\right)$, Eq. (9) is similar to the dispersion relation describing the growth of side bands for a FEL operating at the fundamental. ${ }^{4}$ Although the maximum growth rate found from the dispersion relation (9) is a decreasing function of the bounce frequency $\omega_{b}$ (and thus a decreasing function of the amplitude of the harmonic mode), there is always some value of $m$ for which the growth rate is positive. By approximating the dispersion relation (9) as a quadratic about its minimum, it is straightforward to show that the maximum growth rate of the fundamental mode occurs at $m_{\max }$, where

$m_{\max } \approx-\frac{\omega_{b}(\xi)}{\Omega_{0} \epsilon(\xi)}=-\frac{\left[2 s^{2}\left|C_{s}(\xi) a_{s 0}(\xi)\right|\right]^{1 / 2}}{\Omega_{0} \epsilon(\xi)}$.

This expression is valid as long as $\omega_{b}^{3} \gg C_{1}^{2} / 8$.

Because the parameters in Eq. (9) vary with distance $\xi$, the unstable spectrum of the fundamental mode shifts as the sth harmonic grows. Even though some fundamental modes are always unstable at a particular value of $\xi$, no particular mode is allowed to grow for very long. This is a general feature of harmonic mode suppression and is not limited to FELs, although in a FEL the effect can be enhanced by an appropriate choice of wiggler parameters.

The general approach to achieving maximum suppression is to have $m_{\max }$ (corresponding to the fundamental frequency for maximum growth) change as rapidly as possible, so that no single mode will be unstable for long.

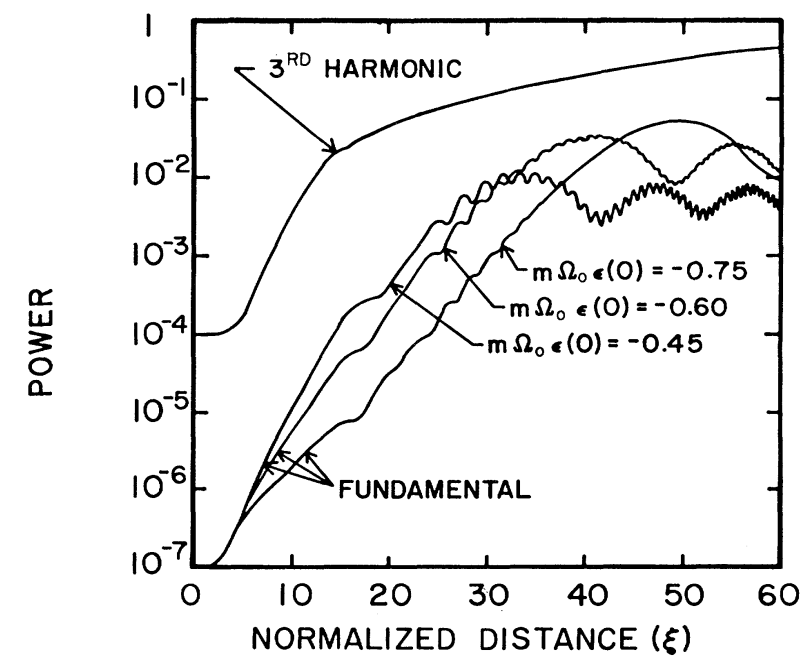

FIG. 1. Power in the harmonic mode and the three fundamental modes with the largest gain vs normalized distance $(\xi)$. The harmonic mode limits the gain of the fundamental to about $10^{5}$, vs about $10^{13}$ when the harmonic is absent.
Since $a_{s 0}(\xi)$ is an increasing function of distance $\xi, m_{\max }$ changes most rapidly if $\left|C_{s}(\xi)\right|$ also increases with $\xi$ and $\epsilon(\xi)$ decreases with $\xi$. The dependence of these parameters on $\xi$ is a function of the type of tapering used. Wwill assume that the period and magnetic field, which determine $\gamma_{r}$ and $a_{w}$, can be tapered independently, although we let $\gamma_{r}(\xi)$ and $a_{w}(\xi)$ have the same functional form:

$$
\begin{aligned}
& \gamma_{r}(\xi)=\gamma_{r}(0)\left[1-\eta_{\gamma}\left(\xi / \xi_{f}\right)^{\rho} \Theta\left(\xi-\xi_{t}\right)\right], \\
& a_{w}(\xi)=a_{w}(0)\left[1-\eta_{a}\left(\xi / \xi_{f}\right)^{\rho} \Theta\left(\xi-\xi_{t}\right)\right],
\end{aligned}
$$

where $\eta_{\gamma}, \eta_{a}, \rho, \xi_{t}$, and $\xi_{f}$ are parameters which we choose $\left(\xi_{t}\right.$ is the position where tapering starts, $\xi_{f}$ is the final value of $\xi$ ), and $\Theta(\xi)$ is the Heavyside step function.

Using the expression for $\gamma_{r}(\xi)$ given in Eq. (11a), the deceleration term $\alpha(\xi)$ becomes

$$
\alpha(\xi)=-\eta_{\alpha} \rho\left(\xi / \xi_{f}\right)^{\rho-1} \Theta\left(\xi-\xi_{t}\right),
$$

where $\eta_{\alpha} \equiv k_{0} L \gamma_{r}(0) S(0) \eta_{\gamma} / \xi_{f}$. Furthermore, if $1+a_{w}^{2}$ $\ll \gamma_{r}^{2}$, which is usually satisfied in practice, the expressions for $C_{n}(\xi)$ and $\epsilon(\xi)$ simplify considerably: ${ }^{1}$

$$
\begin{aligned}
& C_{n}(\xi)=F_{n}\left(a_{w}(\xi)\right)\left[1-\eta_{\gamma}\left(\xi / \xi_{\max }\right)^{\rho} \Theta\left(\xi-\xi_{t}\right)\right]^{2}, \\
& \epsilon(\xi)=\epsilon(0)\left[1-\eta_{\gamma}\left(\xi / \xi_{\max }\right)^{\rho} \Theta\left(\xi-\xi_{t}\right)\right],
\end{aligned}
$$

where

$$
\begin{aligned}
F_{n}\left(a_{w}(\xi)\right) \equiv & \frac{(-1)^{(n-1) / 2}}{2} \frac{a_{w}(\xi)}{a_{w}(0)} \frac{1+a_{w}^{2}(0) / 2}{1+a_{w}^{2}(\xi) / 2} \\
& \times\left[J_{(n-1) / 2}(n x)-J_{(n+1) / 2}(n x)\right],
\end{aligned}
$$

with $x \equiv a_{w}^{2}(\xi) / 4\left[1+a_{w}^{2}(\xi) / 2\right]$ and $n$ odd.

Excluding the frequency resolution $\Omega_{0}$, which is arbitrary, and the harmonic number $s$, for which we chose $s=3$ (third harmonic), the system depends on seven parameters: $a_{w}(0), \eta_{a}, \eta_{\gamma}, \eta_{a}, \rho, \xi_{t}$, and $\xi_{f}$. In our analysis we chose a value of $\rho$ and $a_{w}(0)$, adjusted $\eta_{\alpha}, \eta_{\gamma}$, and $\xi_{t}$ to optimize the gain of the harmonic, and then adjusted $\eta_{a}$ and readjusted $\eta_{\gamma}$ to minimize the gain of the fundamental. We let $\xi_{f}$, the total length of the system in normalized units, be 60 and the initial amplitude of the harmonic, $a_{s}$, be 0.01 . This allowed the harmonic to get well into the nonlinear regime with a tapered section about 5 times longer than the untapered section.

A typical optimized result had $a_{w}(0)=3.0, \rho=2, \eta_{\alpha}$ $=0.24, \eta_{\gamma}=0.05, \eta_{a}=0.50$, and $\xi_{t}=9.0$. For these values, the fundamental mode that reached the largest amplitude $\left[m \Omega_{0} \epsilon(0)=-0.6\right]$ had a gain of around 50 $\mathrm{dB}$, while the gain of the harmonic was slightly greater than $35 \mathrm{~dB}$. A plot showing the harmonic mode and the three fundamental modes with largest gain $\left[m \Omega_{0} \epsilon(0)\right.$ $=-0.45,-0.6$, and -0.75 ] is shown in Fig. 1. From this plot we see that the dominant growth of the fundamental occurs where the harmonic is small; once the amplitude of the harmonic exceeds about 0.2 , the power in the fundamental begins to decrease. Note that the larg- 
est of these three values of $m$ has the largest growth rate and saturates earliest. The overall gain of the fundamental (the power at $\xi=60$ divided by the power at $\xi=0$ ) is about $10^{5}$. While this may seem large, it is significantly smaller than the gain without the harmonic mode present: Simulations with the same parameters but without the harmonic present yielded a gain of about $10^{13}$. Thus, the harmonic reduces the gain of the fundamental by a factor of $10^{8}$.

We now address the issue of harmonic operation versus decreasing the wiggler period; i.e., when increasing the frequency in an FEL amplifier, is it better to operate at the third harmonic or to operate at the fundamental with the wiggler period decreased by a factor of 3? (Recall that the frequency is inversely proportional to the wiggler period.) One consideration is the scaling of the spatial growth rate $k_{i}$ with wiggler period; since the length of the FEL is inversely proportional to the growth rate, too large a reduction in $k_{i}$ will lead to a device that is so long that it becomes impractical.

The scaling of the cold-beam growth rate with wiggler period for an FEL at fixed voltage can be found by combining Eqs. (5)-(8). The analysis is straightforward and in the high-energy limit $\left(\gamma_{r}^{2} \gg 1+a_{w}^{2}\right)$

$$
k_{i} \sim\left(n C_{n}^{2}\right)^{1 / 3}\left(a_{w}^{2} / \lambda_{w}\right)^{1 / 3}\left(1+a_{w}^{2} / 2\right)^{1 / 3}
$$

where $\lambda_{w}=2 \pi / k_{w}$ is the wiggler period at $\xi=0, a_{w}$ is the wiggler parameter at $\xi=0$, and, as above, $n$ is the harmonic number. The first two terms on the right-hand side of this expression represent the standard scaling of gain with wiggler period; ${ }^{5}$ the third term, $\left(1+a_{w}^{2} / 2\right)^{1 / 3}$, is a large-wiggler-parameter effect.

The coupling coefficient $C_{n}$ depends only on $a_{w}$ [see Eqs. (13a) and (14)], so the ratio of the third-harmonic gain to the fundamental gain at one-third the wiggler is

$$
k_{i}\left(n=3, a_{w}\left(\lambda_{w}\right), \lambda_{w}\right) / k_{i}\left(n=1, a_{w}\left(\lambda_{w} / 3\right), \lambda_{w} / 3\right) .
$$

Thus, for a given value of $a_{w}\left(\lambda_{w}\right)$ and $\lambda_{w}$, to make a comparison of the gain we need to know how the wiggler parameter scales with wiggler period. Since $a_{w} \sim \lambda_{w} B_{w}$, where $B_{w}$ is the wiggler magnetic field, the dependence of $a_{w}$ on $\lambda_{w}$ depends on the type of magnet system. Here we consider two cases: the wiggler magnetic field $B_{w}$ independent of $\lambda_{w}$, so that $a_{w} \sim \lambda_{w}$, and $B_{w} \sim \lambda_{w}^{1 / 2}$ for which $a_{w} \sim \lambda_{w}^{3 / 2}$. (For a discussion of the dependence of $B_{w}$ on $\lambda_{w}$, see Refs. 6-8.) In the first case, $B_{w}$ independent of $\lambda_{w}$, when $a_{w}\left(\lambda_{w}\right)=3$ the ratio of the third-harmonic gain to the fundamental gain with reduced wiggler period is about 2. For the second case, $B_{w} \sim \lambda_{w}^{1 / 2}$, the gain ratio is about 3. Thus, in either case a fundamental FEL with the wiggler period reduced by a factor of 3 would be significantly longer than a third-harmonic FEL. We note, however, that at small wiggler parameter $\left[a_{w}\left(\lambda_{w}\right) \lesssim 2\right]$, the conclusion is just the opposite: The gain of a fundamental FEL at reduced wiggler period is larger than the gain of a third-harmonic device. Thus, the size of the wiggler parameter is important when deciding whether to build a harmonic FEL.

A second consideration is thermal spread. In general, the tolerable variation in axial velocity satisfies the constraint $\delta \beta_{z} / \bar{\beta}_{z}<k_{i} / k$, where $k_{i}$ is the spatial growth rate and $k$ is the wave number of the amplified radiation. Thus, when comparing a harmonic FEL and a fundamental FEL operating at the same frequency, the device with the higher gain is less susceptible to thermal spread. As discussed previously, this will be the harmonic FEL for large enough wiggler parameter.

Of course, the total gain of the fundamental in a harmonic FEL also depends on the wiggler parameter and thermal spread; these dependences must be taken into account when designing a realistic device. We have shown that with $a_{w}=3$ and the proper choice of tapering parameters, the fundamental grows in power by a factor of $10^{5}$. If reflectivity low enough to suppress the fundamental can be achieved, then, because of its higher gain, a third-harmonic design at $a_{w}=3$ is superior to a fundamental design with reduced wiggler period. On the other hand, when comparing the effects of thermal spread on the fundamental and the harmonic in the same device, the harmonic gain will be degraded first as thermal spread increases. Thus, it is likely that suppression of the fundamental in a harmonic amplifier requires that the beam be sufficiently cold so that the cold-beam regime is realized.

This work was supported by the Office of Naval Research and the Department of Energy.

${ }^{1}$ W. B. Colson, IEEE J. Quantum Electron. 17, 1417 (1981); N. S. Ginzburg, Zh. Tekh. Fiz. 51, 764 (1981) [Sov. Phys. Tech. Phys. 26, 454 (1981)]; A. K. Ganguly and H. P. Freund, Phys. Rev. A 32, 2275 (1985); H. P. Freund, C. L. Chang, and H. Bluem, Phys. Rev. A 36, 3218 (1987).

${ }^{2}$ S. Benson, D. A. G. Deacon, J. N. Eckstein, J. M. J. Madey, K. Robinson, T. I. Smith, and R. Taber, J. Phys. (Paris), Colloq. 44, C1-353 (1983); B. E. Newman, R. W. Warren, K. Boyer, J. C. Goldstein, M. C. Whitehead, and C. A. Brau, Proc. SPIE 453, 118 (1984).

${ }^{3}$ N. M. Kroll, P. L. Morton, and M. N. Rosenbluth, IEEE J. Quantum Electron. 17, 1436 (1981).

${ }^{4}$ R. C. Davidson and J. S. Wurtele, Phys. Fluids 30, 557 (1987); T. M. Antonsen, Jr., and G. Laval, Phys. Fluids B 8, 1721 (1989).

${ }^{5}$ P. Sprangle, R. A. Smith, and V. L. Granatstein, in Infrared and Millimeter Waves, edited by K. J. Button (Academic, New York, 1979), Vol. 1, p. 279.

${ }^{6}$ R. P. Walker, Nucl. Instrum. Methods Phys. Res., Sect. A 237, 366 (1985).

${ }^{7}$ R. H. Jackson and H. Bluem, in Proceedings of the Eleventh International Conference on Free Electron Lasers, 1989, Conference Digest (IEEE, New York, 1989), p. 146.

${ }^{8}$ W. W. Destler, V. L. Granatstein, I. D. Mayergoyz, and Z. Segalov, J. Appl. Phys. 60, 521 (1986); D. J. Radack and I. D. Mayergoyz, J. Appl. Phys. 67, 4735 (1990). 